\title{
DIMENSIONES DEL RIESGO PERCIBIDO EN EL PROCESO DECISORIO DE ELECCIÓN DE UN PLAN DE SALUD PRIVADO: UN ESTUDIO DE CASO*
}

\author{
LUCIENE EBERLE ${ }^{* *}$, GABRIEL SPERANDIO MILAN*** \& DEONIR DE TONI ${ }^{* * * * *}$ \\ UCS - UNIVERSIDADE DE CAXIAS DO SUL
}

Recibido/ Received/ Recebido: 08/12/2013 - Aceptado/ Accepted / Aprovado: 06/05/2014

\begin{abstract}
Resumen
Este estudio busca identificar las dimensiones del riesgo percibido que pueden ser decisivas en la elección de un plan de salud en el momento de la elección de compra. Se realizó un sondeo aplicado a 216 entrevistados por medio del análisis factorial, el conjunto de 28 atributos se agrupó en cuatro factores, que representaron un índice de explicación de $61,08 \%$. Se concluye que la identificación de las dimensiones del riesgo percibido puede ayudar a las empresas de salud a dar prioridad a acciones, considerando la percepción de los clientes, en el sentido de disminuir la inseguridad y el sentimiento de pérdida en las operaciones de los servicios ofrecidos
\end{abstract}

Palabras clave: Riesgo percibido, Proceso decisorio, Valor percibido, Plan de salud.

\section{PERCEIVED RISK DIMENSIONS IN THE DECISION MAKING PROCESS OF CHOOSING A PRIVATE HEALTH PLAN: A CASE STUDY}

\begin{abstract}
This study seeks to identify perceived risk dimensions that may be decisive for choosing a health plan when purchasing. A survey was applied to 216 respondents through factor analysis, the group of 28
\end{abstract}

Artículo de Investigación científica y Tecnológica derivado del proyecto "Comprobación de los atributos que impactan en el proceso decisorio de elección de un plan de salud privado: un estudio hecho con estudiantes universitarios de administración en Brasil".

* Bacharel em Administração de Empresas (UCS - Universidade de Caxias do Sul), Especialista em Administração Estratégica em Serviços (UCS- Universidade de Caxias do Sul), Mestre em Administração (UCS - Universidade de Caxias do Sul) e Doutoranda em Administração Associação PUCRS/UCS. Professora e coordenadora do curso de pós-graduação Lato Sensu na UCS - Administração Estratégica de Serviços, Estratégias de Marketing e Coordenadora Operacional do curso de Especialização em Planejamento e Gestão de Vendas. Professora do Centro de Ciências da Administração, com ênfase em Administração e Marketing de Serviços, Estratégias de Marketing, Marketing de Relacionamento e Empreendedorismo. E-mail: leberle@ucs.br

*** Bacharel em Administração de Empresas (UCS - Universidade de Caxias do Sul), Especialista em Administração em Marketing (UCS) e em Planejamento Econômico: Gestão Econômica da Empresa (UCS), Mestre em Engenharia de Produção na área de Qualidade e Gerência de Serviços (UFRGS - Universidade Federal do Rio Grande do Sul) e Doutor em Engenharia de Produção na área de Sistemas de Qualidade (UFRGS). Professor, pesquisador e coordenador de Especialização na UCS e no CESF. Experiência na área de Administração, com ênfase em Estratégia Corporativa, Estratégias de Marketing, Comportamento do Consumidor, Marketing de Relacionamento, Satisfação, Retenção, Lealdade de Clientes e Serviços. E-mail: gabmilan@terra.com.br

***** Possui graduação em Bacharel em Filosofia pela Universidade de Caxias do Sul (1992), graduação em licenciado em Filosofia pela Universidade de Caxias do Sul (1992), graduação em Bacharel em Administração pela Universidade de Caxias do Sul (1995), mestrado em Administração pela Universidade Federal do Rio Grande do Sul (1998), doutorado em Administração pela Universidade Federal do Rio Grande do Sul (2005) e Pós-Doutorado em Administração pela Universidade de São Paulo. Tem experiência na área de Administração, com ênfase em Administração de Empresas, atuando principalmente nos seguintes temas Imagem de Produto $e$ Preço, Estratégia de Precificação. E-mail: deonirdt@terra.com.br 
attributes was condensed into 4 factors, which represented a rate of explanation of $61.08 \%$. It was concluded that perceived risk dimensions identification can help healthcare companies to prioritize actions, considering customers perception, in terms of decreasing the uncertainty and loss perception in the services offered.

Keywords: Perceived Risk, Decision making process, Perceived value, Health Plan.

\title{
DIMENSÕES DO RISCO PERCEBIDO NO PROCESSO DECISÓRIO DE ESCOLHA DE UM PLANO DE SAÚDE PRIVADO: UM ESTUDO DE CASO
}

\begin{abstract}
Resumo
Este estudo procura identificar as dimensões do risco percebido que podem ser decisivas na escolha de um plano de saúde no momento da compra. Realizou-se uma sondagem aplicada a 216 entrevistados por meio de análise fatorial. O conjunto de 28 atributos se agrupou em quatro fatores, que representaram um índice de explicação de $61,08 \%$. Conclui-se que a identificação das dimensões do risco percebido pode ajudar as empresas de saúde a dar prioridade a ações que considerem a percepção dos clientes, no sentido de diminuir a insegurança e o sentimento de perda nas operações dos serviços oferecidos.
\end{abstract}

Palavras chave: Risco percebido, Processo decisório, Valor percebido, Plano de saúde.

Eberle, L., Milan, G. \& De Toni, D. (2014) Comprobación de los atributos que impactan en el proceso decisorio de elección de un plan de salud privado: un estudio hecho con estudiantes universitarios de administración en Brasil. En: Revista de la Facultad de Ciencias Económicas de la Universidad Militar Nueva Granada. rev.fac.cienc.econ, XXII (1).

JEL: H51, I12

\section{Introducción}

Actualmente, hay diversos estudios que abordan las dimensiones del riesgo percibido direccionando los esfuerzos para definir un conjunto genérico de dimensiones que sea aplicable para cualquier tipo de empresa, teniendo en cuenta sus especificidades. En general, son considerados cinco tipos de riesgos percibidos en la literatura, siendo ellos: financiero, desempeño (funcional), físico, psicológico y el social (Mitra, Reiss \& Capella, 1999; Goodwin, 2009).

Ante esto, se constata que en los últimos años, la toma de decisión y el riesgo percibido involucrado, han sido discutidos en diversas áreas del conocimiento como filosofía, economía, estadística, psicología y en la gestión. En la administración, estas temáticas son ampliamente discutidas en las investigaciones de comportamiento del consumidor, principalmente, cuando se trata del proceso decisorio de consumo (Tuu, Olsen \& Linh, 2011; Mitchell, 1999).

Sin embargo, se observa que no hay un consenso en la literatura al definirse el riesgo percibido, el cual asume diversas facetas, pero el punto común destacado es que el concepto de riesgo está directamente asociado al comportamiento humano de elección (Dowling, 1986), evidenciando la evaluación general de satisfacción en relación a un determinado producto o servicio (Tuu, Olsen \& Linh, 2011). Partiendo de la definición básica de Solomon (2002), el riesgo percibido está, íntimamente, conectado a la etapa de evaluación y elecciones de alternativas disponibles en un contexto, en el proceso de decisión de consumo. 
Las dimensiones del riesgo percibido influyen directamente en el proceso decisorio de consumo, pues se observa que el mercado actual está repleto de diversas opciones de elección por el hecho de existir un número infinito de productos de consumo. Por lo tanto, este surtido extenso genera una disminución en la motivación en el momento de realizarse la elección, pérdidas de preferencia y satisfacción por la opción elegida, además de un aumento significativo de emociones negativas que incluyen la decepción y el arrepentimiento por la elección realizada (Scheibehnne, Greifeneder \& Todd, 2010; Iyengar, Hubermann \& Jiang, 2004; Chernev, 2003).

Además de eso, el riesgo percibido gana una mayor importancia cuando se trata del consumo de servicios, motivado por la intangibilidad donde el cliente no puede experimentar o probar los servicios a priori (Laroche et al., 2004), donde la confianza se vuelve fundamental en situaciones en que el cliente necesita asumir riesgos, pero no tiene un control completo sobre los resultados esperados (Kim, Ferrin \& Rao, 2008). Siendo así, la intangibilidad y la confianza presentan mayor significado cuando se trata de prestación de servicio.

Entre los diferentes segmentos, la prestación de servicios en salud recibe destaque por sus particularidades que influyen en las definiciones estratégicas y en las prácticas gerenciales. En este contexto, los servicios de salud poseen como característica principal la atención eficaz con la intención de reducir los riesgos percibidos en el momento decisorio de consumo (elección), porque de una forma general, este servicio está siendo consumido por obligación o miedo (Gregório, 2009), reforzando así, la correlación entre consecuencias e inseguridades (Bettman, 1973).

Tratándose de Brasil, se estima que una cuarta parte de la población está afiliada o tiene convenio con algún tipo de plan de salud privado (Bahia \& Luiz, 2006). De acuerdo con eso, Spiller Et al. (2009) refuerza que las estrategias de gestión de una empresa de salud deben estar alineadas con los sentimientos y expectativas de los clientes. El autor destaca, incluso, que los servicios de salud son resultados de experimentaciones (experiencias) y la venta de estos servi- cios están basadas fuertemente en la generación y en la atención a las promesas realizadas a sus usuarios.

Además, es oportuno comentar que, generalmente, los servicios de salud poseen alto contacto entre los prestadores de servicios y los clientes (o usuarios) (Berry \& Pendapudi, 2007; Padma, Rajendram \& Sai, 2009), siendo que no es sólo la experiencia del usuario como paciente que determina la posibilidad de ser retenidos a determinado proveedor de servicios, sino todos los momentos de interacción vividos en los encuentros de servicios entre las partes involucradas. En este sentido, Grönroos (2009) destaca que una empresa proveedora de servicios de salud tiene que superarse en su atención para que pueda generar confianza, compromiso y lazos relacionales, para que los clientes satisfechos puedan crear vínculos duraderos.

\section{Referencial Teórico}

\subsection{Comportamiento del Consumidor y las Elecciones en el Proceso Decisorio}

La toma de decisión de consumo ha sido objeto de estudio tanto en el medio académico como en las prácticas gerenciales en el mundo de los negocios, además de ser bastante investigada en el área de la psicología con la intención de comprender el comportamiento humano. Entonces, conocer cómo los consumidores toman sus decisiones de compra y lo que ellos desean recibir en la utilización y consumo de los productos (bienes tangibles y servicios) es fundamental para que las empresas alcancen el éxito en mercados competitivos (Sehth, Mittal \& Newman, 2001).

De acuerdo con Richers (1984), el comportamiento del consumidor es entendido como estudio de las actividades (procesos) mentales y emocionales, involucradas en la selección, compra y uso de productos / servicios para satisfacer las necesidades y deseos de los consumidores. Diversos autores definen las etapas del proceso de consumo, pero la más citada y utilizada envuelve cinco etapas que son: el reconocimiento del problema, la búsqueda de información, la evaluación (elección) de las ofertas, la compra y la post-compra (Solomon, 2002). 
Sternberg (2000) comenta que la toma de decisión comprende la evaluación (juicio) de oportunidades disponibles y, consecuentemente, la forma de selección entre las opciones disponibles, o sea, al tomar decisiones las personas buscarán maximizar la ganancia (consumo positivo) y minimizar la pérdida (consumo negativo). Este proceso es denominado por el autor como la "teoría de la utilidad subjetiva esperada".

Las elecciones, en el proceso decisorio de consumo, permiten que las personas seleccionen precisamente productos (bienes tangibles e intangibles) que mejor satisfagan sus propias preferencias, dentro de los límites de sus recursos. Además, la elección es vista como esencial para la autonomía, que es absolutamente fundamental para el bienestar. Personas saludables lo quieren y lo necesitan para dirigir sus propias vidas (Markus \& Schwartz, 2010). Además, al hacerse una elección se asume una sobrecarga en relación al número de opciones disponibles para elegir, pudiendo llevar a consecuencias adversas, en caso de que sean hechas elecciones insatisfactorias, generando así, una disminución en la motivación para la elección o la satisfacción con la opción finalmente elegida.

Otro fenómeno de la toma de decisión abordado por algunos investigadores de la psicología, y que tiene un encadenamiento con el consumo (marketing) es el hecho de que, según cómo las opciones son presentadas influyen en la selección de una opción (producto/servicio). Sin embargo, Tversky \& Kahneman (1981) destacan que, cuando los individuos se deparan con una cuestión que envuelve ganancias potenciales, tienden a elegir las opciones que demuestran un menor riesgo, o sea, buscan elegir las opciones que ofrecen una ganancia menor, pero evitando la inseguridad.

Ampliando la discusión, se verifica que hay un énfasis en el desarrollo de estudios que tratan el consumo de productos industrializados, olvidándose del hecho de que el consumo de servicios, debido a sus características exclusivas, abarca diferentes procesos de evaluación por parte de sus consumidores. Por lo tanto, los consumidores tienen un período de evaluación (elección) más complejo, por el hecho de que los servicios son intangibles, no estandarizados y por ser el cliente coproductor (Lovelock \& Wirtz, 2006).

Complementando, Zeithaml \& Bitner (2003) presentan las cuatro categorías de la toma de decisión de los consumidores y evaluación (elección) de los servicios, siendo ellas: (i) la búsqueda de información - que es hecha a través de fuentes personales y riesgo percibido; (ii) la evaluación del conjunto de alternativas - considerando la emoción y el humor involucrados; (iii) la compra y el consumo - la guía del servicio y la compatibilidad de los clientes y ; (iv) la evaluación post- compra, que es el nivel de insatisfacción y la lealtad a la marca.

\subsection{Riesgos percibidos en la prestación de servicios}

Al estudiarse el comportamiento del consumidor y los factores que influyen en los individuos en el momento de hacer sus elecciones, el riesgo percibido emerge como una variable de fundamental importancia en el consumo actual, principalmente, en el consumo de servicios, pues estos son intangibles, sin estándar y, la mayoría de las veces, no tienen garantía y no pueden ser devueltos (Zeithaml \& Bitner, 2003).

Ante esto, se hace necesario comprender la conceptualización de riesgo percibido, lo cual recibió un abordaje en las investigaciones de comportamiento del consumidor a partir del estudio de Bauer (1967). Según Mitchell (1999), el riesgo percibido es un concepto complejo, diferenciado y dinámico, que posee dos componentes: la inseguridad, que tiene relación con el conocimiento previo (Dowling, 1986) y, las consecuencias, que son el grado de importancia o peligro derivado del resultado de la decisión de compra (Bateson, 1995). Complementando tal razonamiento, Feartherman \& Savlou (2003) definen el riesgo percibido a partir del grado de inseguridad y de la importancia del resultado obtenido.

Tanto los investigadores de marketing como algunos psicólogos miden el riesgo subjetivo percibido (Mitchell, 1999; Goodwin, 2009), considerando el hecho de que los consumidores generalmente no saben 


\section{DIMENSIONES DEL RIESGO PERCIBIDO EN EL PROCESO DECISORIO DE ELECCIÓN}

DE UN PLAN DE SALUD PRIVADO: UN ESTUDIO DE CASO

exactamente el riesgo real incluido en sus decisiones (Mitchell, 1999).

La definición más aceptable, según Yates \& Stone (1992) comprende el potencial, la inseguridad y la relevancia de las pérdidas o daños. También se observa que en los estudios de comportamiento del consumidor el riesgo envuelve consecuencias negativas (Stone \& Gronhaug, 1993).

Actualmente, el tema riesgo percibido aborda la diferencia entre lo esperado y los resultados, considerando las expectativas individuales en el proceso decisorio de consumo, así como el grado de importancia atribuido a las pérdidas involucradas. Esto ocurre por el hecho de que al tomar una decisión de compra, el cliente no tiene asegurado, previamente, que obtendrá los resultados esperados, o cuando la medición del resultado sólo puede ser realizada después de la toma de decisión o consumo (Slovic et al., 2004).

Para Mitchell (1999), los gestores organizacionales y los investigadores de marketing han intentado identificar los factores de riesgo percibido considerando cinco motivos a destacar: (i) el riesgo percibido tiene un apelo intuitivo haciendo con que los profesionales tomen decisiones desde el punto de vista de los consumidores; (ii) el riesgo percibido es aplicable a cualquier contexto debido a su gran versatilidad; (iii) el riesgo percibido ayuda en el entendimiento del comportamiento del consumidor, pues estos, tendenciosamente, buscan evitar los errores y maximizar sus compras; (iv) las estrategias de marketing pueden ser direccionadas como reductoras del riesgo y ; (v) el estudio de la percepción de riesgo puede ser una idea.

Sin embargo, se observa que no hay un consenso en la literatura a respecto de las dimensiones de riesgos, considerando las consecuencias de una decisión de consumo, pero la mayoría de los autores considera cinco dimensiones de riesgos que son las siguientes: financiero, físico, social, desempeño y psicológico (Mieres, Martin \& Gutierrez, 2006; Tuu, Olsen \& Linh, 2011).

Según Epstein (1967) las investigaciones sobre el riesgo percibido poseen dos abordajes que son el analítico y el experimental. El abordaje analítico es más subjetivo, pues trata dos hechos formadores de la evaluación del riesgo, compuesta por la inseguridad y las consecuencias (Mandel, 2008). Por otro lado, el abordaje experiencial enfoca, más específicamente, la forma como los individuos obtienen y lidian con el conocimiento, o sea, hay una preocupación con los elementos que interfieren en la evaluación del riesgo percibido. En este abordaje, también, son considerados los elementos externos en relación a los atributos del producto/servicio, como, por ejemplo, el despertar de emociones (Lin, 2008).

Otro abordaje a ser discutido en relación al riesgo percibido, evidencia más específicamente, el discernimiento en el momento del consumo de servicios, como siendo la medición de la inseguridad del desempeño (calidad) antes de la compra efectiva, debido a un nivel reducido de conocimiento $e$ informaciones sobre las operaciones particulares de cada servicio. Ese contexto hace que el consumidor pueda experimentar situaciones de in/satisfacción en encuentros de servicios específicos (Mitra, Reis \& Capella, 1999).

De acuerdo con Chen \& Li (2007), las empresas que desean disminuir el efecto negativo de la percepción de riesgo, deben direccionar sus esfuerzos para consolidar la lealtad del consumidor. Además, las estrategias de comunicación y de promoción, en algunos servicios como, por ejemplo, los de salud y de seguridad deben ser direccionadas para todos los miembros de la familia, y así, aumentar los niveles de confianza. Estas estrategias deben consolidar la confianza del consumidor y dar sustentación a los demás miembros de la familia, buscando reducir los niveles de percepción de riesgos sociales y psicológicos (Tuu, Olsen \& Linh, 2011; Gruber \& Frugone, 2011) facilitando, de este modo, la elección y la decisión de compra.

\subsection{El Impacto de la Confianza y el Valor Percibido en el Proceso Decisorio de Consumo}

La confianza en empresas prestadoras de servicios es altamente significativa por el hecho de que los clientes, en esencia, compran una promesa implícita o explícita de servicio, la cual podrá configurarse 
en el futuro en una experiencia satisfactoria, o no (Claycomb \& Martin, 2001).

La confianza en el proveedor de servicios y en sus colaboradores desenvuelve lazos emocionales entre las partes disminuyendo, así, el riesgo de un desempeño insatisfactorio. Desde el punto de vista relacional, la confianza, según Hewett \& Bearden (2001), es un importante mecanismo que actúa tanto en la persuasión como en el ánimo, demostrando que los resultados serán mutuamente benéficos cuando una parte cree en la integridad del otro y viceversa.

De esta forma, Coulter \& Coulter (2002) destacan la confianza como un factor reductor de inseguridades $y$ de vulnerabilidades, principalmente en las actividades caracterizadas por la intangibilidad y complejidad de naturaleza técnica, como es el caso de los servicios. Los principales beneficios de la confianza son: la reducción de los costos de transacción y la reducción de los riesgos implícitos en los procesos de cambio, contribuyendo para la formación de la retención $\mathrm{o}$, incluso, de la lealtad del cliente y, especialmente, la reducción de los riesgos percibidos, por medio de la percepción de los clientes en relación a la honestidad, a la integridad y a los elevados patrones éticos del proveedor de servicios.

El valor percibido, a su vez, despunta como un constructo que mantiene relación entre lo que el cliente recibe (beneficios) y los esfuerzos (sacrificios) de los que el cliente dispone al adquirir un servicio, considerado como la base de sustentación de las relaciones cliente-empresa, siendo que la preferencia del consumidor es, por lo tanto, consecuencia del valor percibido en sus experiencias pasadas relacionadas a la compra y al consumo (Sirdeshmukh, Singh \& Sabol, 2002).

De acuerdo con Zeithaml \& Bitner (2003), el valor percibido se refiere a la evaluación total del consumidor sobre la utilidad de un servicio, basada en percepciones de lo que es recibido (beneficios) y de los sacrificios en que se incurre para suplir sus necesidades. En esta dirección, las organizaciones deben buscar el entendimiento y la atención del valor esperado por el cliente, para desenvolver relaciones rentables y desde una perspectiva de largo plazo (Weaver, Weber \& Mccleary, 2007).

Por lo tanto, el valor es verificado a partir de la percepción del cliente y, por eso, se ha destacado como contenido integrante de las estrategias empresariales, sumado al concepto de orientación para el mercado, por el cual el enfoque principal es el cliente (Woodruff, 1997).

Tratándose de servicios de salud, el estudio de Chachal \& Kumari (2011), destaca que proporcionar valor agregado a los clientes continúa siendo el objetivo principal, pero refuerzan que los proveedores de servicios de la salud necesitan reconocer cuáles son los elementos de la operación de servicio que pueden presentar mayor riesgo a sus clientes. Complementando, Grönroos (2009) destaca que una empresa proveedora de servicios de salud tiene que superarse en atención para que pueda generar confianza, compromisos y lazos relacionales, además de reforzar sus elecciones en el proceso decisorio de consumo.

\section{Metodología}

\subsection{Problema y objetivos de la investigación}

La definición del problema es la etapa más importante de la investigación, pues solamente mediante la identificación clara y precisa del problema es posible conducir el trabajo de forma adecuada (Malhotra, 2012). Siendo así, el objetivo es la identificación de las dimensiones percibidas por los usuarios de planes de salud privados, relacionados al riesgo percibido, que influyen en el proceso decisorio de elección y consumo de sus servicios.

Para eso, fueron definidos los siguientes objetivos para la investigación: (i) verificar la influencia del riesgo percibido en la intenciones de consumo de servicios por los usuarios de planes de salud; (ii) identificar las dimensiones (factores) que componen el riesgo percibido de los usuarios con respecto a los servicios prestados y; (iii) contribuir con informaciones relevantes, indicando prioridades de acción, en el sentido de mejorar los servicios prestados por los planes de salud privados. 


\subsection{Técnicas y procedimientos de investigación adoptados}

En cuanto al método utilizado, se realizó un investigación cuantitativa a través de un sondeo (Malhotra, 2012) con la aplicación de un cuestionario adaptado de Milan \& Trez (2005), con la intención de medir el riesgo percibido de los usuarios de planes de salud que resultará en las dimensiones (factores) de los riesgos percibidos, a partir de una escala Likert de 7 puntos. Para el tratamiento de los datos fueron utilizadas técnicas estadísticas multivariadas (Hair Jr. et al., 2009; Johnson \& Wichern, 2002).

En la secuencia, a partir de la estructuración del cuestionario de la investigación fue implementada una prueba previa, o prueba piloto, en la cual el instrumento de recolección de datos fue sometido a 10 usuarios de plan de salud privado, con el fin de verificarse la facilidad de comprender las preguntas, de rellenar las respuestas y de posibles mejoras (contenido y forma) a ser incorporadas. Los entrevistados no presentaron dificultades en lo que se refiere a entender y rellenar el cuestionario.

\subsection{Recolección de datos}

El proceso de colecta de datos se ha realizado en el mes de noviembre de 2012, y ha involucrado a estudiantes universitarios de la carrera de Administración de una Institución de Enseñanza Superior (IES) comunitaria, de un universo de 2.000 alumnos. Es importante destacar que al aplicar los cuestionarios fue hecha una breve explicación del instrumento con el intuito de fortalecer la importancia del estudio. Después de aplicados y recogidos, los cuestionarios fueron analizados y procesados.

Todos los cuestionarios recibieron números de control (código), siendo inspeccionados uno a uno, para la digitación de los datos. Con el banco de datos formateado, se realizó una revisión minuciosa y se prosiguió a la preparación de los datos y al análisis estadístico. La muestra final de los cuestionarios válidos, ya excluido los casos de no respondientes o fallas a la hora de rellenarlos, fue de 216 casos.

\subsection{Tratamiento de los datos}

Con el fin de atender a los objetivos de la investigación, los datos obtenidos fueron analizados en una perspectiva cuantitativa. Para eso, se utilizó el método del Análisis Factorial, utilizándose la matriz rotada de correlaciones, también llamado de Varimax con la Normalización de Kaiser, usando el software SPSS (Statistical Package for the Social Sciences) versión 20.0. Este proceso tiene como finalidad que, para cada componente principal, existan apenas algunos pesos significativos y todos los otros sean próximos a cero, a través de la maximización de la variancia entre los factores para la rotación de las matrices factoriales.

Según Aaker, Kumar \& Day (2004), uno de los objetivos del análisis factorial es la combinación de variables que crean nuevos factores o constructos. Esas variables, de acuerdo con Johnson \& Wichern (2002), son agrupadas en función de sus correlaciones. Siendo así, el modelo del análisis factorial estima los factores y las variancias, de modo que las covariancias o las correlaciones previstas estén lo más próximo posible a los valores observados. Para eso, los métodos de estimación o extracción más usados son el de componentes principales y el de la máxima verosimilitud. En este trabajo fue utilizado el método de componentes principales. De esta forma, se objetivó, mediante aplicación de la técnica de análisis factorial, sustituir el conjunto inicial de 30 variables, por un menor número de factores, manteniendo una significativa explicación de las variables originales, de modo a identificar las dimensiones latentes.

\section{Análisis e Interpretación de los Resultados}

\subsection{Caracterización de la Muestra}

A partir de la recolección de datos, se procedió al análisis del perfil de los entrevistados. En cuanto al (género) sexo de los entrevistados que participaron de la investigación, se percibe la predominancia del sexo femenino, representado por $51,76 \%$ de los entrevistados, mientras que las personas del sexo masculino representan $48,24 \%$ de la muestra. En rela- 
ción a la edad, se observó que la media de edad de los entrevistados es de 26 años y ya ocupan cargos o ejercen funciones administrativas a causa de estar cursando una carrera universitaria en el área de gestión.

En cuanto al plan de salud que los entrevistados poseen, se observa que la mayoría de los entrevistados posee Unimed, representando $58,37 \%$ de los entrevistados, mientras los demás planes representan $41,63 \%$ de la muestra. Este índice muy representativo en un único plan se explica por el hecho de que Unimed es un plan de salud a nivel nacional, mientras que los demás planes, a pesar de su tradición, son planes locales y, algunos de ellos no poseen cobertura fuera de la ciudad.

Dando secuencia al análisis de resultados, otro modo de determinar la veracidad del Análisis Factorial es a partir de la prueba de esfericidad de Bartlett y el KMO (prueba Kaiser-Meyer-Olkin) (Hair Jr. et al., 2009). Estas pruebas ofrecen la probabilidad estadística de que la matriz tenga correlaciones significativas entre las variables (Pestana \& Gageiro, 2005). El presente estudio mostró altas correlaciones, siendo que la prueba de esfericidad de Bartlett fue de 3867,173 y encontró un grado de significancia de 0,000 mostrando correlación entre las variables. Además, el KMO presentó un valor próximo a 1 (0,926), lo que indica que el Análisis Factorial es una técnica adecuada para el presente estudio.

Con el intuito de facilitar la interpretación de los factores, fue empleado el método de componentes principales con rotación ortogonal de factores, la rotación Varimax, minimizando el número de variables con cargas altas sobre un factor, reforzando la interpretación de ellos (Malhotra, 2012) por medio de la maximización de las variancias de las cargas de los factores (Johnson \& Wichern, 2002), lo que evidenció que la matriz de correlaciones es adecuada a la técnica de análisis elegida, según datos presentados en la Tabla 1.
Tabla 1. Prueba KMO y de esfericidad de Bartlett ${ }^{1}$

\begin{tabular}{|c|c|c|}
\hline \multicolumn{2}{|c|}{ Prueba de adecuación de la muestra } & Medidas \\
\hline \multicolumn{2}{|c|}{ KM0 (Kaiser-Meyer-Olkin) } & 0,926 \\
\hline $\begin{array}{c}\text { Prueba de } \\
\text { esfericidad de } \\
\text { Bartlett }\end{array}$ & Chi- cuadrado aproximado & 3867,173 \\
& Grados de libertad & 406 \\
\hline
\end{tabular}

Es importante resaltar que fueron consideradas las variables que presentan valor superior a $0,5 \% \mathrm{y}$, por eso, la variable "Servicios complementares ofrecidos (v9)" que presentó un valor de 0,296 y la variable "Entrega del Guía Médico (v21)" con valor de 0,481 fueron excluidas del análisis. Ampliando el análisis y tras la definición de los factores, se adoptó el autovalor (eigenvalue) igual o superior a 1 , sin definición previa del número de factores y cargas factoriales iguales o superiores a 0,30 (Hair Jr. et al., 2009), según los datos presentados en la Tabla 2.

Tabla 2. Factores y los eingevalues ${ }^{2}$

\begin{tabular}{|c|c|c|c|}
\hline \multirow{2}{*}{ Factores } & \multicolumn{3}{|c|}{ Eingevalues } \\
\cline { 2 - 4 } & Total & \% de Variancia & \% Acumulativo \\
\hline 1 & 7,080 & 24,414 & 24,414 \\
2 & 3,932 & 13,559 & 37,973 \\
3 & 3,433 & 11,837 & 49,810 \\
4 & 3,268 & 11,268 & 61,078 \\
\hline
\end{tabular}

A seguir, en la Tabla 3, son presentadas las dimensiones o factores identificados, con sus respectivos atributos y cargas factoriales.

El factor 1 "Competencia y Estructura" representa $24,41 \%$ de la variancia total y está compuesto por once variables positivamente relacionadas. Los atributos que se encontraron en el primer factor (dimensión), pueden ser considerados uno de los más importantes en la percepción de los afiliados, pues se verificó que, es nítida la preocupación con el riesgo físico que enfatiza la importancia de las instalacio-

\footnotetext{
Fuente: Resultados de la investigación.

2 Fuente: Resultados de la investigación.
} 
Tabla 3. Identificación de los factores ${ }^{3}$

\begin{tabular}{|c|c|c|c|c|c|c|}
\hline & Atributos & Factor 1 & Factor 2 & Factor 3 & Factor 4 & Factor 5 \\
\hline $\begin{array}{c}\text { F1 - Competencia y } \\
\text { Estructura }\end{array}$ & $\begin{array}{l}01 \text { - atención de los médicos y empleados } \\
02 \text { - cobertura del plan } \\
03 \text { - competencia de los médicos } \\
08 \text { - precisión de los resultados de los exámenes y } \\
\text { consultas } \\
16 \text { - confort ofrecido al afiliado } \\
17 \text { - equipamientos disponibles } \\
18 \text { - estructura ofrecida al afiliado } \\
19 \text { - limpieza de las instalaciones } \\
20 \text { - ubicación de las instalaciones } \\
23 \text { - imagen transmitida por el plan } \\
28 \text { - confianza en los médicos }\end{array}$ & $\begin{array}{l}0,636 \\
0,625 \\
0,681 \\
0,710 \\
0,561 \\
0,795 \\
0,823 \\
0,856 \\
0,737 \\
0,715 \\
0,569\end{array}$ & & & & \\
\hline $\begin{array}{l}\text { F2 - } \\
\text { Precio }\end{array}$ & $\begin{array}{l}11 \text { - informaciones de las tasas cobradas } \\
12 \text { - forma de reajuste de precios } \\
13 \text { - informaciones sobre reajustes de precios } \\
14 \text { - precio cobrado en relación a otros planes } \\
15 \text { - precio cobrado en relación a los servicios } \\
22 \text { - facilidad de obtener informaciones sobre el plan }\end{array}$ & & $\begin{array}{l}0,582 \\
0,794 \\
0,789 \\
0,668 \\
0,692 \\
0,545\end{array}$ & & & \\
\hline $\begin{array}{c}\text { F3 }- \\
\text { Confianza y Comunicación }\end{array}$ & $\begin{array}{l}24 \text { - informaciones recibidas al afiliarse al Plan } \\
25 \text { - preocupación de la administración del Plan } \\
26 \text { - informaciones sobre cambios en el plan } \\
27 \text { - confianza en los empleados } \\
29 \text { - comunicación con los afiliados } \\
30 \text { - respeto por parte de los empleados }\end{array}$ & & & $\begin{array}{l}0,552 \\
0,562 \\
0,562 \\
0,697 \\
0,641 \\
0,577\end{array}$ & & \\
\hline $\begin{array}{c}\text { F4- } \\
\text { Atención }\end{array}$ & $\begin{array}{l}04 \text { - facilidad para agendar consultas } \\
05 \text { - número de médicos disponibles } \\
06 \text { - número de personas atendiendo } \\
07 \text { - puntualidad de los médicos } \\
10 \text { - tiempo de espera para ser atendido }\end{array}$ & & & & $\begin{array}{l}0,649 \\
0,611 \\
0,533 \\
0,733 \\
0,709\end{array}$ & \\
\hline
\end{tabular}

nes, equipamientos y cuidados con la limpieza. Estos son aspectos que evidencian la tangibilidad de los servicios y que tienen una relación directa con la calidad del servicio prestado (Milan \& Trez, 2005). En lo que se refiere a la competencia, los médicos y demás empleados que forman parte de un plan de salud, son los responsables por demostrar seguridad y confianza a través de su competencia profesional, disminuyendo así, el nivel de inseguridades y riesgo percibido, según destacado por Berry \& Pendapudi (2007). Además, esa dimensión agrupó las variables que destacan la estructura de un plan de salud que

3 Fuente: Resultados de la investigación. 
comprende los hospitales, laboratorios, consultorios y demás espacios físicos, o sea, los aspectos tangibles que son consideradas las variables controlables en servicios de salud y que refuerzan la confianza en las operaciones de los servicios y la dedicación del prestador con su afiliado.

El factor 2 denominado "Precio", representa 13,56\% de la variancia total y está compuesto por seis variables positivamente relacionadas. La segunda dimensión, se refiere a los riesgos financieros que forman la relación de la percepción costo versus beneficios, que agrupó los atributos precio y forma de pago. Se entiende que, de una forma general, los afiliados perciben que reciben un determinado nivel de retorno en contrapartida al valor pagado (mensualidades), disminuyendo la posibilidad de asumir riesgos (Yates \& Stone, 1992). Además, la respuesta del consumidor de servicio de salud en relación al precio depende del tipo de cuidados necesarios y del nivel de contacto con la operadora de salud.

El factor 3, "Confianza", representa $11,84 \%$ de la variancia total y está compuesta por seis variables positivamente relacionadas. Esta dimensión se relaciona directamente al riesgo psicológico percibido, pues confiar es aceptar los riesgos asociados a la profundidad e interdependencia entre las partes involucradas en una relación (Sheppard \& Sherman, 1998; Sirdeshmukh, Singh \& Sabol, 2002). Cabe destacar que, las relaciones entre los profesionales de salud (médicos y enfermeras) y los asociados (y pacientes) deben reflejar una interacción positiva en la operación de servicio. Esta relación en los servicios de salud puede ser entendida como la forma en que el paciente se expone a los profesionales de la salud con la intención de disminuir sus aflicciones e inseguridades.

Por último, el factor 4 (Atención), representa 11,27\% de la variancia total y está compuesta por 4 variables positivamente relacionadas. Esta dimensión despunta como uno de los aspectos principales de la prestación de servicio (Zeithaml \& Bitner, 2003; Grönroos, 2009), y que influye directamente en la toma de decisión y en la evaluación del consumidor, manteniendo una relación directa con los constructos emoción y humor que son muy latentes en servicios de salud (Lin, 2008). La atención se relaciona con el riesgo funcional y que, según Milan \& Trez (2005) evidencian la necesidad de la empresa en calificar su personal con el intuito de mejorar las operaciones de atención al cliente, enfatizando aspectos como agilidad, receptividad y empatía. Además, la comunicación despunta como punto clave en la salud, pues termina siendo el canal de contacto entre el plan de salud y el afiliado en el momento de compartir informaciones esenciales (Spiller et al., 2009). En este factor la comunicación se vuelve esencial para corregir los problemas de forma clara y objetiva (Piva et al., 2007), disponiendo de una relación directa con el riesgo social que tiene como objetivo aproximar a todos los involucrados en la prestación de servicio.

Para evaluar la confiabilidad de las medidas y la consistencia interna de los datos, fue utilizado el coeficiente Alpha de Cronbach (Churchill Jr., 1979; Hair Jr. et al., 2009). Este coeficiente fue desarrollado para calcular la confiabilidad de una prueba en aquellas situaciones en que el investigador no tiene la oportunidad de hacer otra entrevista con los entrevistados, pero que, sin embargo, necesita obtener una estimativa apropiada de la magnitud del error de la medida. Para que el índice sea aceptable, Churchill Jr. (1979) sugieren que los valores sean iguales o superiores a 0,70 , siendo aceptable 0,60 en investigaciones de carácter exploratorio (Hair Jr. et al., 2009). Se percibe que los coeficientes de los cuatro factores variaron de 0,857 a 0,942, siendo considerados satisfactorios.

\section{Consideraciones finales}

Este estudio evidenció la importancia del tema y la escasez de estudios aplicados al contexto de servicios de salud. Frente a eso, el presente trabajo se propuso identificar los factores que influyen en el riesgo percibido en el proceso decisorio de consumo y de elección de la prestadora de servicios de planes de salud privados, con el intuito de direccionar sus esfuerzos para la adecuación a las necesidades y a las expectativas de los afiliados, respaldando las operaciones de gerenciamiento por parte de los gestores. Por consiguiente, fueron identificados cuatro factores del riesgo percibido que son: la competencia y la estructura; el precio; la confianza y comunicación y la atención, con sus respectivos atributos. 
Hace casi una década, algunos autores vienen señalando la importancia de aplicación de diferentes técnicas de gestión en el área de la salud (Enthoven $\&$ Tollen, 2005). Otro aspecto a ser considerado en este sector es la relación que debe ser establecida entre la operadora de planes de salud y los clientes (Piva \& Baptista, 2009) con el objetivo de buscar la continuidad de los contratos que representan la duración de las relaciones, para que consigan alcanzar una mayor rentabilidad de los clientes y de la organización, considerando el nivel de contactos entre el cliente y las empresas prestadoras de servicios, como los servicios de salud que son de alto contacto entre prestador y paciente (cliente).

Por este motivo, se hace necesario entender las necesidades (básicas) y las expectativas generadas por el consumidor de servicios de salud, siendo el punto fundamental para que las operadoras de salud tengan condiciones de adaptar cada etapa de las operaciones del servicio prestado y su nivel de contacto, con la intención de disminuir o amenizar los riesgos percibidos, de acuerdo con lo que el usuario realmente espera y desea.

De acuerdo con la literatura investigada, otro factor evidenciado en este estudio, es el hecho de que el riesgo percibido declina a medida que el beneficio y el valor percibido aumenta (Fischhoff et al., 1978). Eso ocurre porque la oferta de servicios de salud está compuesta por un paquete de beneficios tangibles $e$ intangibles que busca satisfacer las necesidades básicas de los clientes. Una vez que el consumidor hace sus elecciones buscan, al máximo, disminuir las pérdidas y los posibles riesgos.

Además, la satisfacción de clientes en servicios de salud se relaciona directamente con el comportamiento de los clientes en hacer recomendaciones a terceros, con la continuidad y el mantenimiento de los cuidados con la operadora de la salud (Metha, 2011). Por consiguiente, Abiodun (2010) destaca que la satisfacción de clientes deriva de la percepción de la calidad de los servicios a partir de la evaluación de los elementos intangibles asociados a la interacción entre el cliente y el personal del plan de salud durante el tratamiento.
Tratándose de la prestación de servicio de forma general, la toma de decisión de consumo puede incurrir en los más diversos riesgos que son percibidos como acciones $\mathrm{u}$ operaciones que poseen la probabilidad de tener desempeños adversos. Estos resultados negativos pueden resultar en riesgos funcionales, financieros, físicos, psicológicos, sociales e, incluso, de pérdida de tiempo (Mieres, Martin \& Gutiérrez, 2006). Para minimizar el impacto del riesgo percibido, estratégicamente, las prestadoras de servicios deben entrenar a sus empleados y mapear y monitorear sus procesos, con el fin de que los consumidores desarrollen una expectativa adecuada de calidad y satisfacción (Zeithaml \& Bitner, 2003) para que no se arrepientan de la toma de decisión y de sus elecciones realizadas.

\section{Implicaciones gerenciales y limitaciones del estudio}

Se constata que el actual escenario destaca el aumento de la competición entre los planes de salud para atraer y mantener a los afiliados, y por eso, el conocimiento sobre sus preferencias e inseguridades en relación a los servicios prestados puede contribuir a la definición de estrategias para una gestión más efectiva, partiendo del presupuesto de que afiliados satisfechos tienen menor probabilidad de buscar a la concurrencia.

Analizar el comportamiento del consumidor constituye importante fuente de estrategia competitiva de las organizaciones y, en el momento que el abordaje de administración estratégica de las operaciones de servicios pasa a ser relevante también en el ámbito de los planes de salud, estos necesitan conocer los factores que componen los riesgos percibidos para su público específico y maneras de evaluarlos para direccionar sus acciones con el intuito de reducir el riesgo y maximizar los sentimientos de confianza y satisfacción de sus afiliados.

Asociado a eso, los planes de salud necesitan hacer que todos sus colaboradores asuman el compromiso con la imagen de la organización, en todos los aspectos, integrando todos los sectores e unidades, haciendo que los personas que entran en contacto con el afiliado (paciente), formen parte de la solución 
de problemas. Al identificarse factores críticos en la prestación de servicio, que endosan la percepción de riesgo, estos deben ser corregidos, lo más breve posible, evitando que este riesgo se concretice y haga que el cliente experimente niveles de insatisfacción y aumente los índices de deserción de clientes.

Las situaciones prácticas en las organizaciones prestadoras de servicios de salud evidencian la relación entre el entendimiento del comportamiento del consumidor, del formateo de niveles de confianza, de valor percibido y de los riesgos percibidos en las operaciones de los servicios de salud, como factores de empuje de la lucratividad y rentabilidad para alcanzar mayores niveles de sustentabilidad y competitividad organizacional.

A partir de los resultados de la investigación, el trabajo presenta contribuciones relevantes a respecto de los atributos que constituyen los factores que componen el riesgo percibido en el proceso decisorio de elección de una operadora de plan de salud privado, y que deberán ser probadas empíricamente, en el futuro, con un abordaje cualitativo. Una limitación identificada en este estudio es el hecho de que los respondientes son alumnos universitarios, despertando, así, la posibilidad de esta investigación ser aplicada en los clientes-empresas que contratan planes de salud colectivos. Otro campo promisor sería la realización de un sondeo, considerando un plan de salud específico, con el intuito de realizarse un modelaje de los constructos antecedentes y consecuentes del riesgo percibido. Aunque sean contribuciones inherentes a una prestación de servicio que posee muchas especificidades, existe la posibilidad de ampliarse a la discusión y de replicarse investigaciones similares en otros contextos, buscando disminuir la sensación de pérdida inherente al riesgo percibido, facilitando así, el proceso de decisión y haciendo que las elecciones sean satisfactorias.

\section{Referencias}

Aaker, D. A., Kumar, V. \& Day, G. S. (2004). Pesquisa de marketing (2. ed.) São Paulo: Atlas.

Abiodun, A. J. (2010). Satisfaction with quality attributes of primary health care services in Nigeria. En: Journal of Health Management, XII (1): 39-53.
Bahia, L. \& Luiz, R. R. (2006). Informações sobre coberturas e preços de planos e seguros privados de saúde: as perspectivas da complementaridade entre fontes administrativas e de base populacional. En: Ciência \& Saúde Coletiva, XI (4): 878-880.

Bateson, J. (1995). Managing Services Marketing: text and readings (3. ed.) Orlando: The Dryden Press.

Bauer, R. A. (1967). Consumer Behavior as Risk Taking. En: Cox, D. (Ed.) Risk Taking and Information Handling in Consumer Behavior. Harvard University, Boston Graduate School of Business Administration: 23-33.

Berry, L. L. \& Bendapudi, N. (2007). Health Care: a fertile Field for service research. En: Journal of Service Research, X (2): 111-122.

Bettman, J. (1973). Perceived risk and its components: a model and empirical test. En: Journal of Marketing Research, X: 184-190.

Chahal, H. \& Kumari, N. (2011). Evaluating customer relationship dynamics in healthcare sector through indoor patients' judgement. En: Management Research Review, XXXIV (6): 626-648.

Chen, M. F. \& Li, H. L. (2007). The consumer's attitude toward genetically modified foods in Taiwan. En: Food Quality and Preference, XVIII: 662-674.

Chernev, A. (2003). When More Is Less and Less Is More: the role of ideal point vailability and assortment in consumer choice. En: Journal of Consumer Research, XXX (2): 170-183.

Churchill Jr., G. A. (1979). A paradigm for developing beter measures of marketing constructs. En: Journal of Marketing Research, VXI: 64-73

Claycomb, C. \& Martin, C. L. (2001). Building customer relationships: an inventory of service providers' objectives and practices. En: Marketing Intelligence \& Planning, XIX, (6/7): 385-399.

Coulter, K. S. \& Coulter, R. A. (2002). Determinants of trust in a service provider: the moderating role of length of relationship. En: Journal of Services Marketing, XVI (1): 35-50.

Dowling, G. (1986). Perceived Risk: the concept and its measurement. En: Psychology \& Marketing, III (3): 193-210.

Enthoven, A. C. \& Tollen, L. A. (2005). Competition in healthcare: it takes systems to pursue quality and efficiency. En: Health Affairs, W5: 420-433.

Epstein, S. (1967). Integration of the cognitive and the Psychodtnamic unconscious. En: American Psychologist, XXVII (5): 385-392.

Featherman, M. S. \& Pavlou, P. A. (2003). Predicting e-services adoption: a perceived risk facets perspective. En: International Journal of Human-Computer Studies, 59: 451-474.

Fischhoff, B., Slovic, P., Lichtenstein, S., Read, S. \& Combs, B. (1978). How safe is safe enough? A psychometric study of attitudes towards technological risks and benefits. En: Policy Sciences, IX: 127-152.

Goodwin, S. A. (2009). The concept and measurement of perceived risk: a marketing application in the context of the new product development process. En: Proceedings of ASBBS Annual Conference, Las Vegas, XVI (1).

Gregório, R. (2009). Marketing Médico: criando valor para o paciente. Rio de Janeiro: Editora DOC.

Grönroos, C. (2009). Marketing, gerenciamento e serviços (3. ed.) Rio de Janeiro: Elsevier.

Gruber, T. \& Frugone. F. (2011) Uncovering the desired qualities and behaviours of general practitioners (GPs) during medical (service recovery) encounters. En: Journal of Service Management, XXII (4): 491-521. 


\section{DIMENSIONES DEL RIESGO PERCIBIDO EN EL PROCESO DECISORIO DE ELECCIÓN \\ DE UN PLAN DE SALUD PRIVADO: UN ESTUDIO DE CASO}

Hair Jr., J., Anderson, R., Tatham, R. \& Black, W. (2009). Análise multivariada de dados" (6. ed.). Porto Alegre: Bookman.

Hewett, K. \& Bearden, W. O. (2001). Dependence, trust, and relational behavior on the part of foreign subsidiary marketing operations: implications for managing global marketing operations. En: Journal of Marketing, 65 (4): 51-66.

Iyengar, S. S., Huberman, G. \& Jiang, W. (2004). How Much Choice Is Too Much? Contributions to 401(k) Retirement Plans. En:"Pension Design and Structure: New Lessons from Behavioral Finance, Oxford: Oxford University Press: 83-95.

Johnson, R. A. \& Wichern, D W. (2002). Applied multivariate statistical analysis (5. ed.). Upper Saddle River: Prentice Hall.

Kim, D. J., Ferrin, D. L. \& Rao, H. R. (2008). A trust-based consumer decision-making model in electronic commerce: the role of trust perceived, risk and their antecedents. En: Decision Suport Systems, 44: 544-564.

Laroche, M., Mcdougall, G., Bergeron, J. \& Yang, Z. (2004). Exploring how intangibility affects perceived risk. En: Journal of Service Research, VI (4): 373-389.

Lin, W. B. (2008). Investigation on the model of consumers perceived risk: integrated viewpoint. En: Expert Systems with Applications, 34: 977-988.

Lovelock, C. \& Wirtz, J. (2006). Marketing de Serviços: pessoas, tecnologias e resultados (5. ed.). São Paulo: Pearson Prentice Hall.

Malhotra, N. K. (2012). Pesquisa de marketing: uma orientação aplicada (6. ed.). Porto Alegre: Bookman.

Mandel, D. R. (2008). Violations of coherence in subjective probability: a representational and assessment processes account. En: Cognition, 106: 130-156.

Markus, H. R. \& Schwartz, B. (2010). Does Choice Mean Freedom and Well-Being?: Journal of Consumer Research, 37 (2): 344-355.

Mehta, S. (2011). Service quality as predicator of patient satisfaction: a study of the health care sector. En: Journal of Health Management, XIII (n): 211-239.

Mieres, C.G., Martin, A. M. D. \& Gutierrez, J. A. T. (2006). Antecedents of the difference in perceived risk between store, brands and national brands. En: European Journal of Marketing, 409 (1/2): 61-82.

Milan, G. S. \& Trez, G. (2005). Pesquisa de Satisfação: um modelo para planos de saúde. En: RAE-eletrônica, IX (2).

Mitchell, V. W. (1999). Consumer Perceived Risk: conceptualisations and models. En: European Journal of Marketing, 33 (1/2): 163-195.

Mitra, K., Reis, M. \& Capella, L. (1999). An examination of perceived risk, information search and behavioral intentions in search, experience and credence services. En: The Journal of Services Marketing, XIII (3): 208-228.

Padma, P., Rajendram, C. \& Sai, L. P. (2009). A conceptual framework of service quality in healthcare: perspectives of Indian patients and their attendants. En: Benchmarking: An International Journal, XVI (2): 157-191.
Pestana, M. H. \& Gageiro, J. N. (2005). Análise de dados para ciências sociais (4. ed.). Lisboa: Silabo.

Piva, L., Fumagalli, L., Baptista, P. \& Silva, W. (2007). Relação entre satisfação, retenção e rentabilidade de clientes no setor de planos de saúde. En: Revista de Ciências da Administração, IX (19): 54-80.

Piva, L. \& Baptista, P. (2009). Relação entre a adoção de práticas de promoção da saúde e prevenção de doenças e a percepção de valor para consumidores de plano de saúde. En: XXXIII Encontro Anual da Associação Nacional dos Programas de PósGraduação em Administração (ANPAD). São Paulo: ENANPAD.

Richers, R. (1984). O enigmático mas indispensável consumidor: teoria e prática. En: Revista de Administração, XIX (3): 46-56.

Scheibehenne, B., Greifeneder, R. \& Todd, P. M. (2010). Can there ever be to many options? A Meta-Analytic review of choice overload. En: Journal of Consumer Marketing, 37 (3): 409-425.

Sheppard, B. H. \& Sherman, D. M. (1988). The grammars of trust: a model and general implications. En: Academy of Management Review, XXIII (3): 422-437.

Sheth, J. N., MittalL, B. \& Newman, B. I. (2001). Comportamento do Cliente. São Paulo: Atlas.

Sirdeshmukh, D., Singh, J. \& Sabol, B. (2002). Consumer trust, value, and loyalty in relational exchanges. En: Journal of Marketing, 66 (1): 15-37.

Slovic, P., Finucane, M. L., Peters, E. \& MacGregor D. G. (2004). Risk as analysis and risk as feelings: some thoughts about affect, reason, risk and rationality. En: Risk Analysis, XXIV (2): 311-322.

Solomon, M. (2002). O Comportamento do Consumidor. Porto Alegre: Bookman.

Spiller, E. S., Senna, A. M., Santos, J. F. \& Vilar, J. M. (2009). Gestão dos Serviços de Saúde. Rio de Janeiro: Editora FGV.

Sternberg, R. J. (2000). Psicologia Cognitiva. Porto Alegre: Artmed.

Stone, R. N. \& Gronhaug, K. (1993). Perceived Risk: further considerations for the marketing discipline. En: European Journal of Marketing, XXVII (3): 39-50.

Tuu, H., Olsen, S. \& Linh, P. (2011). The moderator effects of perceived risk, objective knowledge and certainty in the satisfaction-loyalty relationship. En: Journal of Consumer Marketing, XXVIII (5): 363-375.

Tversky, A. \& Kahneman, D. (1981). The framing of decisions and the psychology of choice. En: Science, v. 211, 453-458.

Weaver, P. A., Weber, K. \& McCleary, K. W. (2007). Destination evaluation: the role of previous travel experience and trip characteristics. En: Journal of Travel Research, v. 45 (3), 333-344.

Woodruff, R. (1997). Customer value: the next source for competitive advantage. En: Journal of the Academy of Marketing Science, XXV (2), 139-153.

Yates, F. \& Stone, E. (1992). The Risk Construct. In F. Yates (Org.). Risk-taking behavior. Chichester: Wiley.

Zeithaml, V. A. \& Bitner, M. J. (2003). Marketing de serviços: a empresa com foco no cliente. Porto Alegre: Bookma 
